\title{
Autosomal recessive cutis laxa syndrome revisited
}

\author{
Éva Morava*,1, Maïlys Guillard ${ }^{2}$, Dirk J Lefeber ${ }^{2}$ and Ron A Wevers ${ }^{2}$
}

\author{
${ }^{1}$ Department of Paediatrics, Radboud University Nijmegen Medical Centre, Nijmegen, The Netherlands; ${ }^{2}$ Laboratory of \\ Paediatrics and Neurology, Radboud University Nijmegen Medical Centre, Nijmegen, The Netherlands
}

The clinical spectrum of the autosomal recessive cutis laxa syndromes is highly heterogeneous with respect to organ involvement and severity. One of the major diagnostic criteria is to detect abnormal elastin fibers. In several other clinically similar autosomal recessive syndromes, however, the classic histological anomalies are absent, and the definite diagnosis remains uncertain. In cutis laxa patients mutations have been demonstrated in elastin or fibulin genes, but in the majority of patients the underlying genetic etiology remains unknown. Recently, we found mutations in the ATP6VOA2 gene in families with autosomal recessive cutis laxa. This genetic defect is associated with abnormal glycosylation leading to a distinct combined disorder of the biosynthesis of $\mathrm{N}$ - and O-linked glycans. Interestingly, similar mutations have been found in patients with wrinkly skin syndrome, without the presence of severe skin symptoms of elastin deficiency. These findings suggest that the cutis laxa and wrinkly skin syndromes are phenotypic variants of the same disorder. Interestingly many phenotypically similar patients carry no mutations in the ATP6V0A2 gene. The variable presence of protein glycosylation abnormalities in the diverse clinical forms of the wrinkled skin-cutis laxa syndrome spectrum necessitates revisiting the diagnostic criteria to be able to offer adequate prognosis assessment and counseling. This paper aims at describing the spectrum of clinical features of the various forms of autosomal recessive cutis laxa syndromes. Based on the recently unraveled novel genetic entity we also review the genetic aspects in cutis laxa syndromes including genotype-phenotype correlations and suggest a practical diagnostic approach.

European Journal of Human Genetics (2009) 17, 1099-1110; doi:10.1038/ejhg.2009.22; published online 29 April 2009

Keywords: cutis laxa; wrinkled skin; congenital disorders of glycosylation; gerodermia osteodysplastica; De Barsy Syndrome; ATP6V0A2

\section{Introduction}

Cutis laxa is an acquired or inherited skin disorder characterized by wrinkled, inelastic skin. Inborn errors of elastin synthesis and structural defects of extracellular matrix proteins have been described leading to the decreased elasticity and redundant, sagging skin in patients. ${ }^{1}$ The inherited form of cutis laxa is rather

*Correspondence: Dr É Morava, Department of Paediatrics, Radboud University Nijmegen Medical Centre, P.O. Box 9101, 6500 HB Nijmegen, The Netherlands.

Tel: + 31243619 470; Fax: + 31243616 428,

E-mail: E.Morava@cukz.umcn.nl

Received 6 August 2008; revised 22 December 2008; accepted 8 January 2009; published online 29 April 2009 uncommon. Some 200 families have been reported in literature until now. The majority of the various subtypes of cutis laxa syndromes (Tables 1 and 2) affect connective tissue development through structural gene defects. In most of the patients there is a strong skin-histological evidence demonstrating an abnormal structure of elastin fibers and the associated extracellular matrix network. ${ }^{1}$ Recently autosomal recessive cutis laxa syndrome type II (ARCL II; MIM 219200) emerged in the center of attention, due to the discovery of a unique underlying metabolic etiology. ${ }^{4}$ This novel inborn error is a distinct form of the Congenital Disorders of Glycosylation (CDG). The defect is responsible for the phenotype in several patients diagnosed with the most common and viable form of the cutis laxa 
Table 1 Genetic background in inherited cutis laxa syndromes

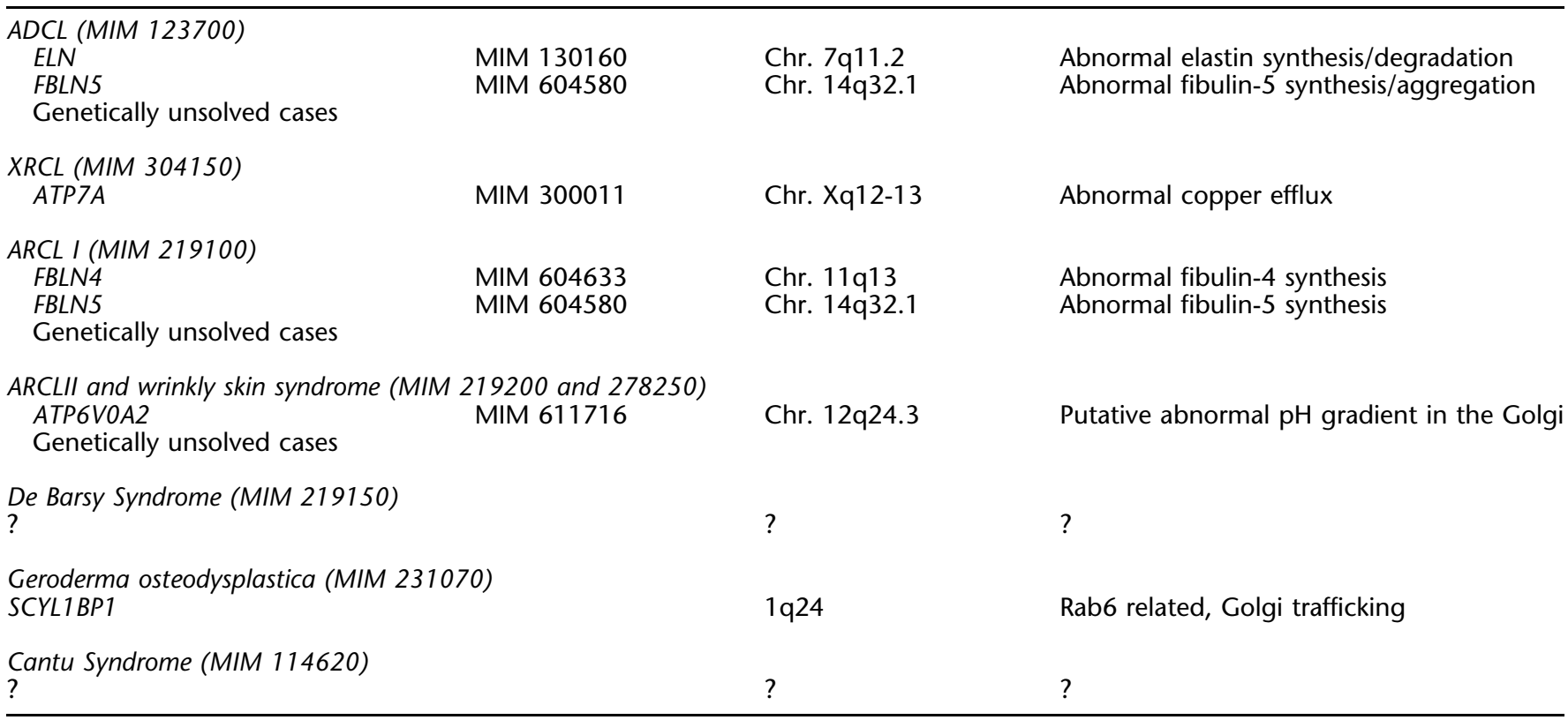

Table 2 Distinctive features in different recessively inherited syndromes with cutis laxa

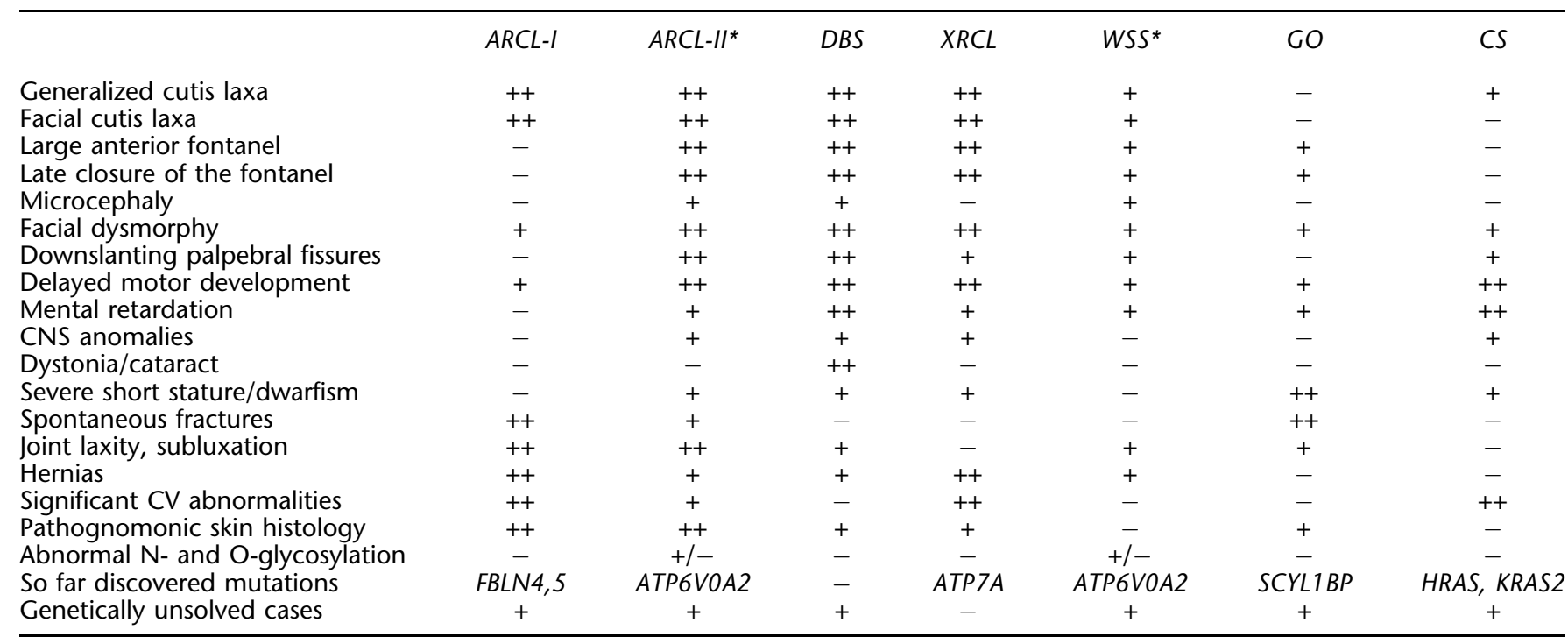

++: characteristic finding; +: feature described; -: not present; *: cases with normal and abnormal glycosylation are both included. ${ }^{2,3}$ ARCL: autosomal recessive cutis laxa; DBS: De Barsy Syndrome; XRCL: X-linked recessive cutis laxa; WSS: wrinkled skin syndrome, GO: geroderma osteodysplastica, CS: Costello Syndrome; CNS: central nervous system; CV: cardiovascular.

disease spectrum..$^{2-4}$ The underlying genetic defect is in one of the subunits of a V-ATPase (ATP6VOA2, MIM $\left.611716^{2,3}\right)$. Abnormal glycosylation of several plasma proteins has been demonstrated in affected patients. This defect is thought to possibly affect glycan biosynthesis and processing by influencing the $\mathrm{pH}$ gradient in the Golgi apparatus. Glycan biosynthesis occurs in many cell types and glycosylation is an essential post-synthetic modification for many intracellular proteins and for secreted proteins. The V-ATPase defect may lead to a multisystem metabolic disease by causing abnormal $\mathrm{N}$ - and O-linked glycosylation. ${ }^{4,2,3}$ It has been hypothesized that the defect 
affects tissue elasticity through altered cellular trafficking and abnormal elastin synthesis (personal communications, Professor Z Urban). Glycosylation is essential in fetal development as well. Therefore, it is not surprising that some patients with a combined $\mathrm{N}$ - and O-glycan biosynthesis defect present with a congenital developmental disorder of the central nervous system. Here, we describe the spectrum of clinical and metabolic features found in various forms of autosomal recessive cutis laxa, and revisit the diagnostic criteria (Table 2). We review the genetic aspects in ARCL, evaluate genotype-phenotype correlation to be able to offer adequate prognosis assessment and counseling and suggest a practical diagnostic approach.

\section{Clinical spectrum in cutis laxa syndromes}

The term cutis laxa implies a specific skin anomaly, with loose, redundant skinfolds and abnormal elasticity of the skin, occurring both as an inherited and acquired condition. ${ }^{5}$ The clinical diagnosis is relatively easy in most patients; however, Ehlers-Danlos Syndrome are frequently considered in the differential diagnosis due to the presence of excessive, wrinkled skin and increased joint laxity. The major clinical difference however is a decrease in elasticity in the former, and hyperelasticity in the latter diagnostic group.

The inborn types of cutis laxa have a diverse genetic background including autosomal dominant (ADCL; MIM 123700), autosomal recessive (ARCL; MIM 219100, MIM 219200, MIM 219150, MIM 231070) and X-linked inheritance (XRCL; MIM 304150). In the autosomal dominant form of cutis laxa syndrome skin and connective tissue symptoms are frequently apparent already at birth or in early childhood. The presence of excessive skinfolds and loose, redundant skin leads to early diagnosis. Associated features include cardiac valve anomalies and hernias. Several familial cases are known with variable long-term outcome. Aortic root dilatation might result in lifethreatening aortic dissection throughout the clinical course. Although early emphysema occurs, gastrointestinal symptoms are quite uncommon. Histological studies are frequently pathognomonic, demonstrating sparse, fragmented elastic fibers in skin. ${ }^{1,6-8}$ Some patients with skin, pulmonary and vascular system involvement and an autosomal dominant type of cutis laxa syndrome carry elastin gene $(E L N)$ mutations. ${ }^{7}$

Patients with an X-linked cutis laxa syndrome have a distinct, unique presentation at birth. Besides generalized cutis laxa they have a thin face, long philtrum, hooked and beaked nose, brittle hair, high forehead and large fontanel, giving a distinct facial appearance. Systemic involvement includes failure to thrive due to chronic diarrhea, malabsorption, congenital hydronephrosis, urethral and bladder diverticulae. Skeletal anomalies consist of large and late closing fontanel, narrow chest, pectus carinatum, coxa valga, short tubular bones, pelvic exostosis, kyphosis and platyspondyly. The specific sign of occipital horn exostoses of the skull is diagnostic for the disease. Cardiovascular symptoms, including orthostatic hypotension, tortuous carotid arteries and easy bruising have been described in some of the patients as well. ${ }^{9} \mathrm{X}$-linked cutis laxa syndrome is allelic to Menkes disease and is caused by mutations in the copper transporter gene ATP7A..$^{9-11}$

Several autosomal recessive forms of cutis laxa are known, but most cases are categorized in the so-called ARCL I (MIM 219100) and ARCL II (MIM 219200) group. The clinical spectrum of the ARCL autosomal recessive cutis laxa syndromes is highly heterogeneous, both with respect to organ involvement and severity. ${ }^{5,12}$ ARCL I is a specific, life-threatening disorder with organ involvement, lung atelectesias and emphysema, diverticulae of the gastrointestinal and genitourinary system and vascular anomalies (arterial aneurysms, fibromuscular artery dysplasia and stenoses). Associated cranial anomalies, late closure of the fontanel, joint laxity, hip dislocation and inguinal hernia have been observed, but uncommon. Diminution of elastic fibers throughout the dermis and abnormal elastin components by electron microscopy are pathognomonic. ${ }^{13-15}$ There is a phenotypic overlap between the very mild form of the recessive forms of cutis laxa, and the autosomal dominant form, which might impede adequate counseling. Although mutations have been detected in the fibulin-5 (FBLN5) and the fibulin-4 (FBLN4, EFEMP2) genes in a few children with the severe, mostly lethal autosomal recessive form of cutis laxa syndrome, the underlying genetic etiology in the majority of cases is still unknown. ${ }^{13,14-16}$

Autosomal recessive cutis laxa syndrome type II appears to be a spectrum of clinical entities with variable severity of cutis laxa, abnormal growth and developmental delay and associated skeletal abnormalities. The classical phenotype includes generalized, loose, redundant skin-folds, with slow return on stretching. The facial skin is less affected. Persistent wide fontanels, frontal bossing, slight oxycephaly, downward slanted palpebral fissures, reversed-V eyebrows and dental caries are characteristic. Findings like intrauterine growth retardation, hip dislocation, pigeon breast, scoliosis, inguinal hernia and flat feet are common. ${ }^{12,17}$

The wrinkled skin syndrome (WSS, MIM 278250 ${ }^{18,19}$ ) appears to be the mild phenotypic manifestation of ARCL type II. The skin biopsy is not pathognomonic for cutis laxa in most WSS patients. Microcephaly and late closing fontanel are characteristic features in both entities; however, these appear in the X-linked recessive form of cutis laxa ${ }^{9}$ and in another autosomal recessive syndrome similar to ARCL II in the De Barsy Syndrome (DBS; MIM 219150 20) as well. Cantu Syndrome (MIM 114620) is a variant of WSS with osteochondrodysplasia and cardiac anomalies. ${ }^{21}$ 
A subgroup of patients with ARCL type II and also some of the patients with WSS carry ATP6VOA2 gene mutations and have an inborn error of metabolism of $\mathrm{N}$ - and O-glycan biosynthesis due to an abnormal proton gradient in the Golgi apparatus and causing a unique glycosylation defect. $^{2,3}$

The De Barsy Syndrome, one of the most intriguing forms of ARCL presents with several features overlapping with ARCL II, including skin symptoms, facial features, cranial abnormalities and growth delay. The disorder is frequently progressive, associated with dystonia, eye anomalies (cataract and corneal abnormalities) and progeroid features. ${ }^{20}$ The skin biopsy is characteristic, showing frayed and reduced elastic fibers. The underlying pathomechanism and the genetic background have not been unraveled yet.

Another, somewhat similar clinical entity, gerodermia osteodysplastica (GO; MIM 231070 ${ }^{19,22,23}$ ) has been previously considered an allelic variant of ARCL type II. So far no glycosylation defect and no ATP6VOA2 mutations could be demonstrated in patients with GO. Recently mutations have been discovered in SCYL1BP1 in a large consanguineous family from Oman and several unrelated cases diagnosed with GO. SCYL1BP1 is a golgin, interacting with Rab6 and associated with the Golgi apparatus and involved in the secretory pathway. ${ }^{23}$ Besides the similar cellular localization of the underlying defects there are several overlapping findings like wrinkled skin and spontaneous fractures but also a significant phenotypic difference between ARCL type II and GO patients; the presence of severe osteoporosis with early spontaneous fractures, significant dwarfism, the lack of large open fontanels and the progeroid features are distinctive for gerodermia osteodysplastica. $^{22}$ There is an evolving phenotype in both syndromes, but in GO the dysmorphic features might get more prominent through the disease course in contrast to the improving phenotype in other forms of ARCL type II.

Wrinkled skin has been described in patients diagnosed with William's Syndrome, and elastin mutations have been described in children with cutis laxa syndrome, without the classical features of William's Syndrome (MIM 194050). In pseudoxanthoma elasticum (MIM 264800) redundant, non-elastic skin changes appear later in the course of the disease. A novel subtype (MIM 610842) appears with coagulation anomalies due to mutations of the $\gamma$-glutamyl carboxylase (GGCX) gene. ${ }^{24}$ Wrinkled, aged looking skin is present in Hutchinson Gilford Syndrome (MIM 176670) with a strikingly characteristic progeroid phenotype due to Lamin A mutations, and in Barber Say Syndrome (MIM 209885) in association with genital anomalies and hypertrichosis. Genital anomalies are also present in combination with skeletal dysplasia in the extremely rare SCARF Syndrome (MIM 312830). In several other genetic syndromes like Costello Syndrome (MIM 218040) or Kabuki Syndrome (MIM 147920) loose skin resembling cutis laxa was noted as an associated feature, mostly with sporadic occurrence. $^{25,26}$ Several case reports describe unique associations of symptoms with wrinkled skin or cutis laxa with an apparent autosomal recessive inheritance, including cortical blindness, ${ }^{27}$ hearing loss, ${ }^{28}$ cleft palate, ${ }^{29}$ leukodystrophy $^{30}$ or hypothyroidism. ${ }^{31}$

\section{Population data}

Cutis laxa syndromes appear to be rather infrequent; however no reliable data on the incidence of inherited cutis laxa have been reported so far. Reviewing the medical literature more than 100 families are described with the 'classical' presentations of autosomal dominant or recessive inheritance of syndromal cutis laxa. The largest group consists of children with an apparent autosomal recessive inheritance, with most of the patients ( $>50$ children) demonstrating a clinical presentation comparable to ARCL II, and a similar number of patients (approximately 40) with an apparent ARCL type I. Autosomal dominant cutis laxa has been reported less frequently with an estimated 20 families. Although wrinkly skin syndrome appears to be more frequent than ARCL type II in the clinical practice, there are less clinical reviews available. The frequency of gerodermia osteodysplastica and XRCL appears to be comparable with the frequency of ADCL based on the number of reports. ${ }^{10,18,22,23}$ It is difficult to assess the frequency of XLCL due to the phenotypic overlap with Menkes Syndrome. ${ }^{9}$ In addition to these numbers several patients have been reported with a syndromic appearance associated with cutis laxa. Summarizing these data, we have to conclude that inherited cutis laxa syndromes are rare, and the number of families reported so far is approximately 200 .

Strikingly only a handful of patients with the different subtypes have been solved genetically. Patients with XLCL Syndrome have been reported carrying mutations in the ATP7A gene. ${ }^{20,21}$ Ten families have all been reported with ADCL and distinct mutations (ELN and FBLN5 $\left.{ }^{6,27}\right)$ and 17 families are known with ARCL II carrying ATP6VOA2 mutations (Professor Z Urban, St Louis, US; personal communication). The success for finding mutations is relatively low in ARCL I patients ${ }^{6,7,14}$ and estimated less than $10 \%$ (Table 1 and 2).

\section{Clinical features in ARCL type II}

Patients with ARCL type II have a distinct clinical phenotype, and can be divided in two major groups (Figure 1): children with ARCL type II associated with a combined $\mathrm{N}$ - and O-linked glycosylation defect (CDG type II; Figure 4), and children without the presence of the metabolic disorder. So far, we have not found specific clinical features distinguishing the metabolic and 

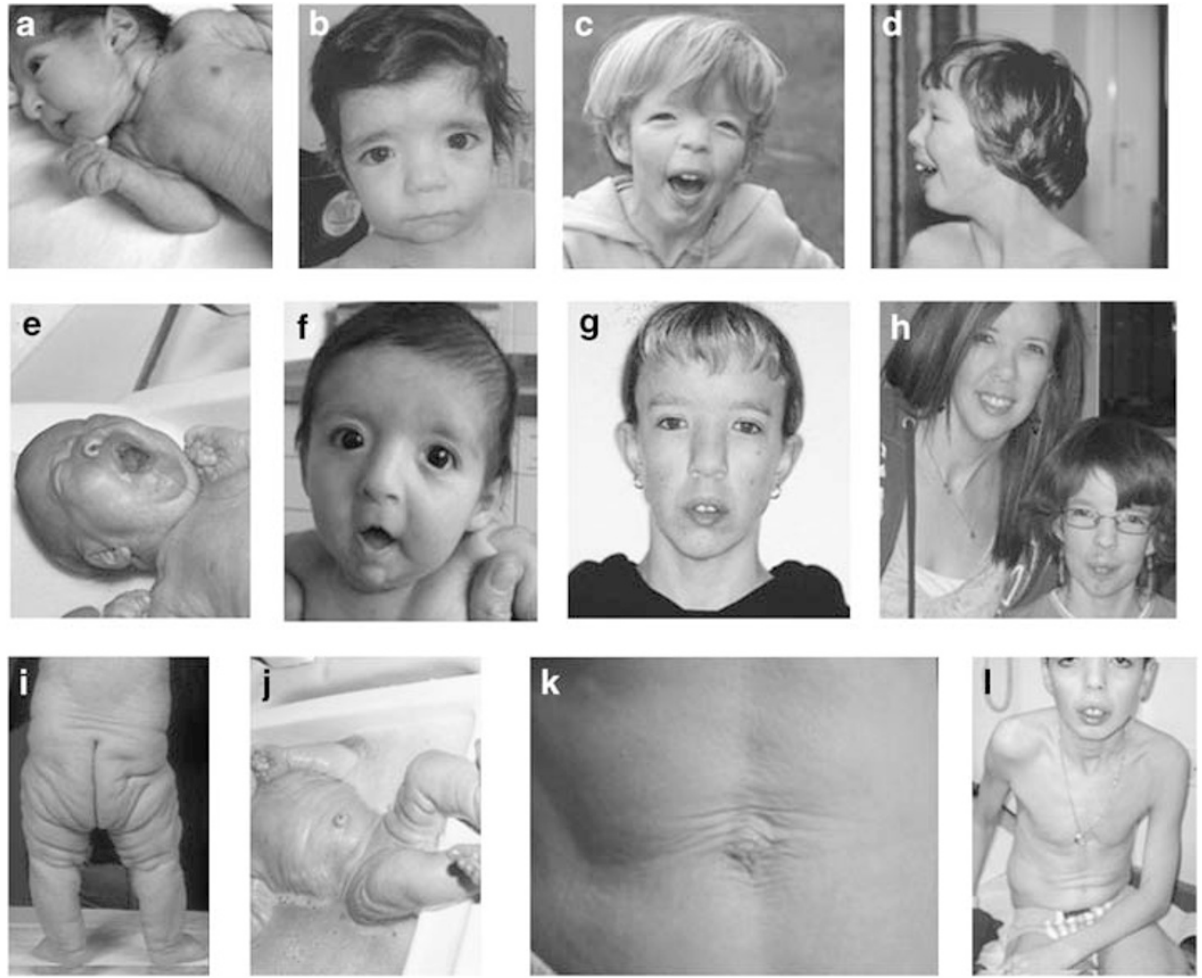

Figure $1(\mathbf{a}-\mathbf{h})$ The characteristic facial features (dyscrania due to open fontanel, down-slanting palpebral fissures, posterior-rotated, prominent ears, long philtrum, short, broad nose) of six patients with ARCL II at different ages (a and f) patient $5^{*}$ at neonatal and 1 year of age, (b) patient $2^{*}$ at 1.5 years, (c and $\mathbf{h}$ ) patient $1^{*}$ at 3 and 13 years of age, (d) patient $9^{*}$ at 11 years of age, $(\mathbf{e}, \mathbf{g}$ and $\mathbf{h})$ patient $6^{*}$ at 20 years. $\left({ }^{*}\right)$. (i $\left.\mathbf{i}-\mathbf{l}\right)$ Skin anomalies with abnormal fat distribution and improving cutis laxa in four patients with ARCL II at different ages (i) patient $7^{*}$ at 1.5 years of age, (j) patient $6^{*}$ at 1 year of age (k) patient $3^{*}$ at 5 years of age and (I) patient $8^{*}$ at 14 years of age $\left({ }^{*}\right.$ : Patient numbers refer to the paper of Kornak et $\left.a^{3}\right)$.

non-metabolic group from each other. Apparently the frequency of central nervous system malformations is somewhat higher in the group with CDG defect, but congenital brain malformations have been described in the non-metabolic group as well. So far, all ARCL II cases manifesting the combined glycosylation defect harbor mutations on both alleles of the ATP6VOA2 gene. In the non-metabolic group the underlying genetic defect is not yet known. ${ }^{3,22}$

The ARCL II patients with CDG type II, described so far, have severe generalized cutis laxa, skeletal, neuromuscular and central nervous system involvement, mental retardation and a combined deficiency in the biosynthesis of $\mathrm{N}$ - and O-linked glycans (Table 2). In addition, we evaluated seven children presenting with a clinical phenotype of generalized cutis laxa at birth. All patients were born at term, and no abnormalities were observed during the pregnancy. All patients had normal growth parameters and presented with generalized cutis laxa at birth. Skin biopsy demonstrated abnormal, broken, shortened and fuzzy elastic fiber structure with a significantly decreased amount of elastin by histology. No corneal abnormality, corneal dryness or cataract was found. None of the patients had symptoms of lung disease, chronic diarrhea, vocal cord laxity, hoarse voice, vascular anomalies, hernias or gastrointestinal anomalies. One child had a complex cardiac anomaly. Half of the patients had congenital bilateral hip dislocations. During the variable follow-up period none of the patients developed spasticity or dystonia. Ophthalmologic evaluation showed a strabismus, myopia ( $<-5$ diopters) or amblyopia with hypermetropia in various patients. The skin anomalies diminished with age. Most children had no apparent fat distribution anomalies at birth but with the improvement of the loose, hanging, hypoelastic, wrinkled skin the abnormal fat distribution around the buttock and the hips became more and more apparent. The systemic involvement was very mild, including elevated liver enzyme activities and slightly abnormal coagulation in some of the patients. No apparent hypoglycemia, endocrine, cardiac or renal involvement has been noted in the patients. Severe pulmonary and cardiovascular involvement were absent in our patients. Congenital microcephaly was observed in most of the patients, in others it progressed further during the course of the disease. In addition to the previously reported migration defects in two of the three children observed in $2005^{4}$ cobblestone-like cortical anomalies were found in other patients (Figure 2). Congenital 

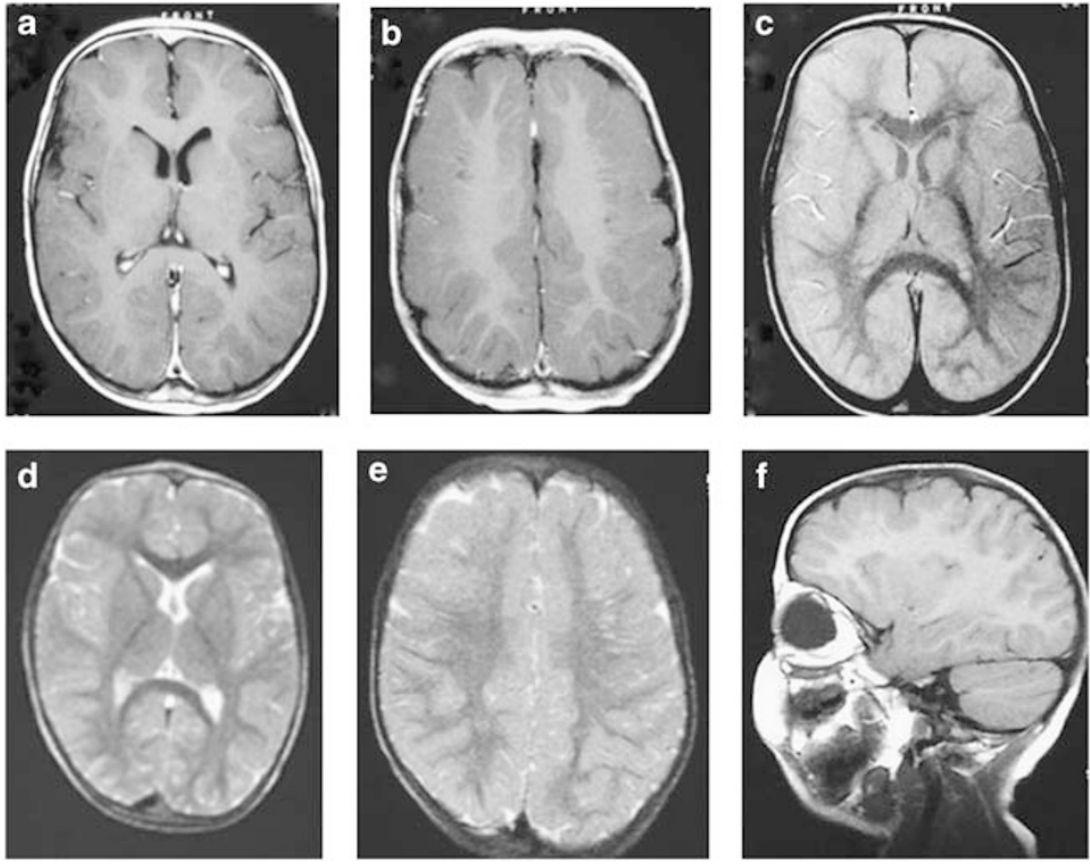

Figure 2 Central nervous system anomalies on MRI T2-weighed images in two patients demonstrating cobblestone-like brain dysgenesis (a-f) (Patient 9 and 10 in the paper of Kornak et $a l^{3}$ ).

central nervous system anomalies, including cerebellar malformations, however, were found only in the minority of the patients. Epilepsy (late onset seizures) occurred in one out of the seven children described here. In the total number of 10 patients sensorineural hearing impairment was noted only in one child. Developmental delay was observed in the majority of the children, partially due to muscle hypotonia, and in some degree hyperflexible joints. Progressive microcephaly is quite unique in the view of the finding of a large and persistent open fontanel. Muscle hypotonia can lead to secondary delay in motor skill acquisition. In view of the frequent occurrence of microcephaly, the mild-moderate developmental delay is most likely central in origin. Mental retardation was present in most older cases in our patient cohort. ${ }^{2}$

\section{Central nervous system involvement in ARCL type II}

Autosomal recessive cutis laxa often occurs in combination with microcephaly, developmental delay and hypotonia. Central nervous system malformations, however, are relatively uncommon. ${ }^{32}$ Brain migration defects on the other hand are pathognomonic for several forms of the disorders of O-glycosylation (O-mannosylation defects). In the heterogeneous clinical group of congenital disorders of $\mathrm{N}$-glycosylation, cerebellar and vermis anomalies are frequent, but migration defects have not been reported yet. ${ }^{33}$ The unique combination of cutis laxa syndrome with congenital brain malformations, analogous to a cobblestone-type brain dysgenesis has been observed in several children with $\mathrm{N}$ - and O-glycosylation defects. ${ }^{4,2,3,34}$

Children with O-mannosyl glycan biosynthesis defects characteristically have congenital muscular dystrophy (CMD) characterized by a decreased $\alpha$-dystroglycan staining in the muscle tissue. ${ }^{35}$ Some of the known $\alpha$-dystroglycanopathies, like Fukuyama muscular dystrophy, muscle-eye-brain disease or Walker-Warburg Syndrome are multiple malformation syndromes associated with brain migration defects and congenital eye anomalies. Defective biosynthesis of O-mannosyl glycans leads to muscle disease by hypoglycosylation of the $\alpha$-dystroglycan and central nervous system developmental defects through disrupted cell interactions with the ECM and abnormal receptor signaling in the embryonic period. ${ }^{36}$ The underlying etiology of the development of the ophthalmologic abnormalities in O-mannosyl glycan disorders is not fully understood.

In spite of the clinical similarities with O-mannosyl glycan defects, the muscle histology in patients with ATP6VOA2 defect shows no signs of a muscle dystrophy, but a mild myopathy. ${ }^{37}$ The most common ophthalmologic findings include strabismus and myopia, and the visus is normal in nearly all cases. ${ }^{34}$ The migration defects leading to cortex dysgenesis (Figure 2) 2, $^{2,3}$ in ATP6VOA2 mutations could be associated both with normal 
intelligence or severe mental retardation. The combination of cobblestone-like brain dysgenesis with late closure of the fontanel and microcephaly might suggest an underlying metabolic (glycosylation) abnormality in children with cutis laxa, but migration defects have been described in children with ARCL type II without a glycosylation defect (Dr L van Maldergem, Liege, Belgium; personal communication).

\section{Histology in autosomal recessive cutis laxa syndromes}

The classical definition of cutis laxa syndromes relies on the detection of abnormal elastin structure by histology. Normal elastic tissue is not visible by hematoxylin-eosin stain by light microscopy, and specific elastin stains must be used to visualize elastic fibers. An even more informative method is to use electron microscopy, demonstrating the microfibrillar structure around an amorphous core in normal elastic fibers. In the initial definition of cutis laxa a combination of light microscopic and electron microscopic findings was used to characterize the classical findings: small and diminished elastic fibers, deficient elastin with globular appearance and tangled microfilaments, excessive microfibrils with abnormal orientation and fragmentation of the elastin structure. ${ }^{21}$

Electron microscopy is diagnostic in most types of autosomal recessive cutis laxa, especially in the severe forms of ARCL I and II demonstrating a moth-eaten appearance, abnormal elastin fiber branching and lose microfibrils by reduced elastin synthesis. In ARCL type I and II increased vascularization in the dermis, reduced collagen bundle size, underdeveloped elastic fibers distinguish ARCL type II from wrinkled skin syndrome. Besides the characteristic frayed elastic fibers, reduced in number and density, the skin collagen fibril network could be fully normal. Several patients with the classic clinical phenotype of De Barsy Syndrome have been reported with normal skin biopsy, but in others reduced density of elastic fibers or abnormal elastin fiber synthesis were observed. ${ }^{2,20,38}$ The presence or absence of generalized elastin fiber anomalies by histology are not part of the diagnostic criteria in patients with gerodermia osteodysplastica. ${ }^{22}$ Even more surprising in autosomal dominant forms, in the presence of an ELN mutation, some individuals could present with mild abnormalities, such as decreased amount of elastic fibers seen by electron microscopy or without histologic alterations. The histology therefore is an important, but not indispensable tool in the diagnostics. One should conclude that the absence of the pathognomonic histology does not rule out the diagnosis of the various disorders in the cutis laxa syndrome spectrum and histology cannot be used to differentiate between the non-metabolic forms and those with combined $\mathrm{N}$ - and O-glycosylation defects. Cutis laxa syndromes should be therefore defined as inherited disorders of lose, hanging skin with decreased elasticity independent from the presence or absence of the histological alterations.

\section{Pathophysiological aspects in ARCL type II patients with a ATP6V0A2 defect}

As described above, a large number of families with ARCL type II or WSS ${ }^{2,22}$ with a distinct phenotype showed lossof-function mutations in the a2-subunit of the V-type $\mathrm{H}^{+}$-ATPase. The $\mathrm{V}$-ATPase complex is composed of a transmembrane V0 domain and a cytoplasmic V1 domain (Figure 3). ${ }^{39}$ The complex is involved in $\mathrm{pH}$ regulation in more or less all acidified intracellular compartments including the Golgi apparatus. The V1 domain has eight subunits A-H and is responsible for ATP hydrolysis. The V0 domain contains six subunits. It takes care of proton translocation and serves as a membrane anchor. ${ }^{39,40}$ The a2-subunit has been described to reside in endosomes and in a compartment overlapping with the trans-Golgi network. $^{41}$ The a-subunit is embedded in the membrane where it serves as an anchor for the large V-ATPase protein complex and is directly involved in proton transport. ${ }^{42}$ Depending on the a-isoform present (either a1-a4 in the V0 domain), the complex will be located in different organels. The design of the four a-isoforms seems to determine $\mathrm{pH}$ differences between the Golgi (a2-isoform) and the more acidic lysosome (a3-isoform). In the transGolgi network of the secretory pathway, located most closely to the glycosylation machinery, the complex most likely contains the a2-subunit and possibly the a1-subunit. The a-subunit has a hydrophobic carboxy-terminus
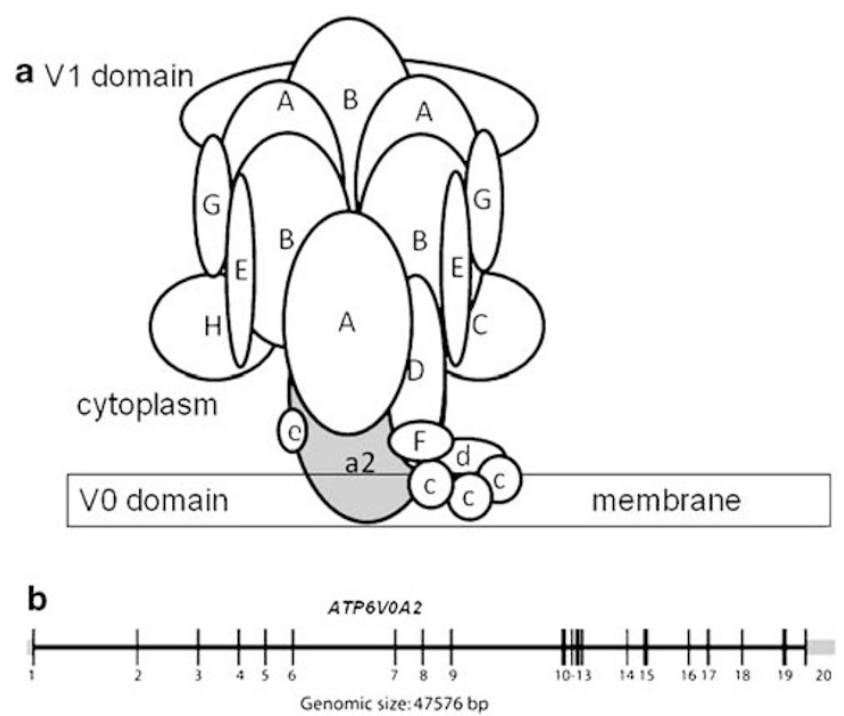

Figure 3 (a) Schematic structure of the V-ATPase complex (based on and adapted from Qi et $a l^{39}$ ) (b) Gene structure of ATP6VOA2. 
containing 8-9 transmembrane helices. The aminoterminal part is hydrophilic and resides on the cytoplasmatic side of the membrane. ${ }^{39}$ An additional role of the V0 domain of the V-ATPase complex has been described in vesicular transport processes in the secretory pathway.

Evidence exists regarding the correlation of V-ATPase dysfunction with abnormal protein glycosylation as observed in ARCL II patients. Neutralization of the intracellular $\mathrm{pH}$ has been shown to impair glycosylation by a disturbance of the location of glycosyltransferases in the secretory route. ${ }^{43}$ As one of the major functions of the V-ATPase is to maintain the slight acidic gradient along the secretory pathway in which protein glycosylation occurs, it could be speculated that also in patient fibroblasts, a change in $\mathrm{pH}$ exists with a disturbed glycosylation as a result. Functional evidence for defective protein transport, a second function of the V-ATPase, has been identified in patient fibroblasts ${ }^{3}$ showing a delayed translocation of Golgi membranes to the ER upon Brefeldin A treatment. This indicates disturbed Golgi-ER retrograde transport, a similar mechanism that has been shown to be abnormal in patients with defects in the Conserved Oligomeric Golgi (COG) complex also having a glycosylation disorder. Although a link between V-ATPase and glycosylation can be identified, it remains to be elucidated how abnormal glycosylation can lead to the complex phenotype of skeletal and skin symptoms associated with central nervous system anomalies.

Patients with defective protein glycosylation are commonly grouped in the CDG, a growing class of hereditary disorders. ${ }^{44,45}$ Although most of the CDG Syndromes are caused by loss of function mutations in glycosyltransferases, it has recently been discovered that mutations in the COG complex can disturb glycosylation and cause disease. ${ }^{44,46-48}$ The function of this complex is in the transport of proteins along the secretory route and COG-deficient patients show a disturbed Golgi-ER retrograde trafficking, a similar finding as has been found for the glycosylation-deficient ARCL II patients. Both groups of patients show a combined disorder of N-glycosylation and mucin type O-glycosylation. Interestingly, Wu et al ${ }^{49}$ described a phenotype of wrinkled skin in COG7-deficient patients. Patients with defects in other subunits of this complex (COG4 and COG8) did not show skin abnormalities.

Isoelectric focusing studies of blood plasma was the initial evidence for an underlying glycosylation defect in some patients with ARCL II. ${ }^{4,2}$ In patients with these abnormal profiles Kornak et $a l^{3}$ identified the underlying ATP6VOA2 gene defect. Figure 4 shows the characteristic plasma isoelectric focusing profiles. Diagnostic screening for abnormal glycosylation involves the isoelectric focusing of plasma transferrin (TIEF) for detection of $\mathrm{N}$ glycosylation disorders and isoelectric focusing of plasma apolipoprotein CIII (Apo C-III) for defects in the core 1 mucin type O-glycan biosynthesis (Figure 4$){ }^{4}$
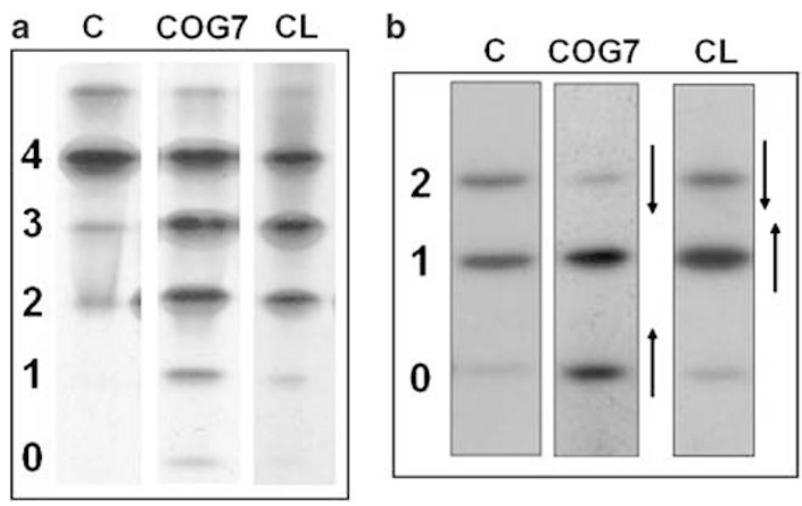

Figure 4 (a) Plasma transferrin isofocusing ( $\mathrm{N}$-glycosylation screen). (b). Plasma Apolipoprotein C-III isofocusing (mucin type Oglycosylation screen). Characteristic profiles for: $\mathrm{CL}$, cutis laxa patients with the ATP6VOA2 defect; COG7, patients with a defect in subunit-7 of the COG complex; C, controls.

The combination of the two biochemical tests offers a simple and reliable diagnostic approach in ARCL type II patients. In patients with the ATP6VOA2 defect transferrin isofocusing shows a characteristic disialo type II profile, whereas Apo C-III isofocusing shows a typical Apo C-III ${ }_{1}$ profile characterized by elevated levels of the Apo CIII-1 isoform and decreased levels of Apo C-III 2 . In young patients with the ATP6VOA2 defect ( $<6$ months), the abnormal Apo $\mathrm{C}-\mathrm{III}_{1}$ profile was found whereas the transferrin isofocusing was normal. In all patients with the ATP6VOA2 defect, including the ones where first the transferrin isoform profile was normal, the typical abnormal profiles for Apo C-III and transferrin were found after the age of 6 months.

\section{Genetic aspects of ATP6VOA2 and related ATPase genes}

Kornak et $a l^{3}$ have described loss of function mutations in the ATP6VOA2 gene encoding the a2-subunit of the V-type $\mathrm{H}^{+}$-ATPase in several patients with ARCL type II. Interestingly, the strongest expression of the gene product is detectable in liver and kidney, organs, which are not specifically involved in the phenotype. The ATP6VOA2 gene is mapped to chromosome 12 (12q24.31). The human ATP6VOA2 gene consists of $47576 \mathrm{bp}$ and is organized in 20 coding exons (Figure 3b). The a2-subunit (Q9Y487) contains 856 amino acids. The ATP6VOA2 gene harbors mutations in both consanguineous ARCL II families and in sporadic cases. ${ }^{2,3}$ In 12 families Kornak et $a l^{3}$ found 10 different mutations causing loss of protein function.

$\mathrm{H}^{+}$-transporting ATPases are ubiquitous in nature; V-ATPases pump protons against an electrochemical gradient, whereas F-ATPases reverse the process, synthesizing ATP. The crucial role of V-ATPases has been demonstrated in several inborn errors, leading to various metabolic disorders associated with internal organ, or 
skeletal abnormalities, dysmorphic features, kidney disease, hearing loss, mental retardation and developmental delay. Mutations in the ATP6B1 gene, encoding the B-subunit of the apical proton pump mediating distal nephron acid secretion, cause distal renal tubular acidosis associated with sensorineural deafness (MIM 267300). Furthermore, several different ATP6VOA4 mutations (MIM 605239) have been described in patients with renal tubular acidosis. $^{50}$

Another example of a similar defect has been described in infantile malignant recessive osteopetrosis (MIM 259700). ${ }^{51}$ Osteopetrosis is caused by a defect in osteoclast function, degrading bone material in a tightly sealed extracellular compartment that is acidified by a vacuolar (V)-type $\mathrm{H}^{+}$-ATPase. OC116 is a gene encoding the a3subunit of the V-ATPase from osteoclasts regulating the acidification machinery. Homozygous truncating loss of function mutations and splice site mutations have been observed leading to disruption of the normal N-terminal related interaction with other subunits of the proton pump. One should note that none of these patients had any skin manifestation of their disease.

Mutations in another member of the ATPase family, in the ATP7A gene were identified in families with Menkes disease and as well in patients with X-linked cutis laxa syndrome. ${ }^{9}$ Studies in cultured cells localized the protein product to the final compartment of the Golgi apparatus, the trans-Golgi network. The protein supplies copper to the copper-dependent enzymes as they migrate through the secretory pathway. So far more than 150 point mutations and deletions are known in the ATP7A gene. Most of these mutations were found to lead to the classic form of Menkes disease, and a few to the milder XLCL Syndrome. ${ }^{10}$

\section{Metabolic disorders with wrinkled skin or cutis laxa}

The first metabolic disorder associated with cutis laxa was Menkes disease. Menkes disease and XLCL are related disorders of copper transport. Various mutations of the ATPA7 gene lead to the two separate (however somewhat overlapping) syndromes. Menkes Syndrome is more severe, presenting with abnormal neurodevelopment, connective tissue problems, and often premature death. The gene encodes a highly evolutionarily conserved, copper-transporting P-type ATPase. The gene normally is expressed in nearly all human tissues, and it localizes to the trans-Golgi network. 9

The second group of patients described with wrinkled skin and metabolic defects included twins with dysmorphic features, severe liver disease and early lethality. Both neonates had excessive skinfolds in the neck and axial regions, and wrinkled skin of the hands and feet. Increased activities of lysosomal enzymes were found in plasma, and total sialic acid in plasma was strongly decreased. Isoelectric focusing of serum sialotransferrins showed a type 2 CDG pattern. O-Glycosylation was investigated by isoelectric focusing of apolipoprotein C-III. It showed increased fractions of hyposialylated isoforms. In a consecutive study a defect in the Conserved Oligomeric Golgi complex was established at the level of subunit COG7, leading to disruption of multiple glycosylation functions of the Golgi. ${ }^{49}$ The same alterations in association with axial cutis laxa were noted in other patients harboring the common COG7 mutation as well. ${ }^{48}$

These reports were followed by several other reports on defects in COG7 but also in other COG subunits (COG4 and COG8) leading to abnormal N- and O-linked glycosylation. ${ }^{46-48}$ However, no skin abnormalities were observed in the other subtypes.

Transaldolase (TALDO) deficiency (MIM 606003) is a newly recognized metabolic disease, reported in two patients with liver failure and cirrhosis. In a family with four infants born to the same consanguineous parents all children presented at birth with dysmorphic features, cutis laxa and hypertrichosis, hepatomegaly, splenomegaly, liver failure, hemolytic anemia, thrombocytopenia and genitourinary malformations. The clinical courses were variable, including early lethality. TALDO activity was undetectable in the patients' tissues, and mutation in the TALDO1 gene was found in the four patients. ${ }^{52}$ No glycosylation abnormalities could be confirmed in the patients, and the etiology of cutis laxa remained so far unknown (Dr V Valayannopoulos, Paris, France; personal communication). As cutis laxa has not been described in other TALDO patients the finding may be coincidental and due to the consanguinity in this family.

\section{Conclusions}

ARCL is heterogeneous and may occur both with and without glycosylation abnormalities. In our patient group demonstrating the characteristic clinical features and the biochemical phenotype of CDG type II we detected ATP6VOA2 mutations in all of the patients. It is rather intriguing that many children with ARCL II present with glycosylation anomalies, and others with the same phenotype do not show any metabolic alteration, supporting genetic heterogeneity in AR cutis laxa syndrome. ${ }^{53}$ The phenotypic variability regarding growth and development in different patients could be the consequence of consanguinity. However, these findings underline even more the usefulness of glycosylation studies in the diagnosis of ARCL II.

All of the children described so far with ATP6VOA2 mutations have a combined disorder of glycosylation with a characteristic TIEF pattern (CDG type II) and an abnormal isofocusing profile for core 1 mucin type O-glycan in blood, demonstrated by the abnormal results of the Apo C-III IEF. The pattern is different from the severe hypoglycosylation observed in COG gene defects, ${ }^{36,47}$ but 


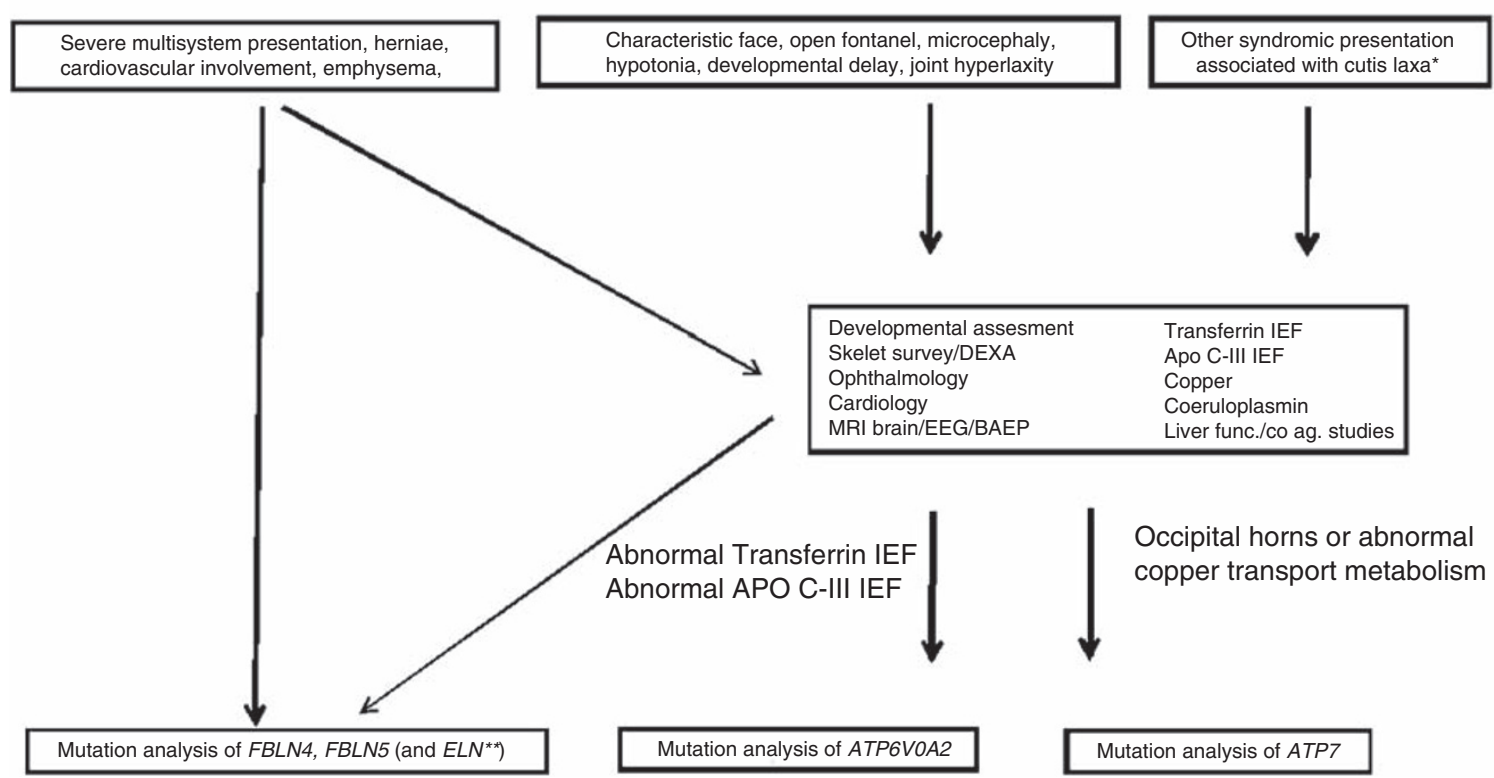

Figure 5 Diagnostic flowchart in inherited cutis laxa (*Rule out Costello Syndrome in case of clinical suspicion; ${ }^{* *}$ Dominant cases might phenotypically overlap).

there is an obvious increased level of the ApoC $\mathrm{III}_{1}$ fraction and decreased levels of Apo C- $\mathrm{III}_{2}$.

The use of glycosylation analysis in patients with various forms of cutis laxa facilitates the further diagnostic steps including mutation analysis (Table 2, Figure 5). One should emphasize the possibility of missing the diagnosis of CDG Syndrome in young infants with congenital cutis laxa by screening for glycosylation disorders by the TIEF method. In some of the children the results of the TIEF were normal at the age of 4 months and became diagnostic only months later. Fortunately, the Apo C-III IEF pattern was abnormal at an early stage of the disease, leading to the correct diagnosis. We suggest evaluating the protein glycosylation status in all children with congenital wrinkled skin or cutis laxa, especially with characteristic facial features, late closure of the fontanel, variable central nervous system involvement and developmental delay.

Categorization and further sub-typing of ARCL Syndromes gets rather difficult due to evolving phenotype in many children with vanishing skin abnormalities especially after puberty. Moreover, with the same mutations the phenotype may be variable. Although there are no therapeutic strategies available in ARCL accurate diagnosis is of pivotal importance for natural history postulations and genetic counselling. For the clinicians involved (pediatricians, dermatologists, clinical geneticists) it is difficult to come to a correct diagnosis. The availability of a simple biochemical test in serum for the ATP6VOA2 defect changes the diagnostic approach implying a screen for congenital disorders of glycosylation in all patients with a congenital cutis laxa syndrome as a first diagnostic approach. We suggest a simple and practical diagnostic approach for the workup of patients with a suspected autosomal recessive cutis laxa (Figure 5). Table 2 summarizes the major clinical difference between the most common types of autosomal recessive syndromes associated with cutis laxa (Table 2). ARCL I patients have a striking presentation with systemic involvement and lifethreatening organ symptoms. Patients with ARCL type II and children with WSS have generalized skin abnormalities and a large, open fontanel, commonly associated with microcephaly. There are many overlapping features regarding the phenotype in De Barsy Syndrome and ARCL II. Although both groups show a late closure of the anterior fontanel, the facial features are quite different, demonstrating a somewhat older, almost progeroid appearance in De Barsy Syndrome. Other diagnostic signs such as athetoid movements and corneal opacification can distinguish DBS from both ARCL II and ARCL I (Table 2). XRCL patients have distinct facial appearance, distal cutis laxa and develop 'occipital horns'. Most patients with gerodermia osteodysplastica present with milder skin symptoms and spontaneous fractures are already in the first months of life. The distribution of cutis laxa (hand and feet), the characteristic face, peri-oral papillomata, and the presence of pulmonary stenosis facilitates recognition of Costello Syndrome. Skin histology might confirm the diagnosis in ARCL type I and type II; however, could be fully normal in 
the other types, therefore cannot be used for differentiation in many forms.

In case of a new patient presenting with generalized cutis laxa or wrinkled skin (Figure 5) we suggest performing a two directional skull $\mathrm{X}$ ray (fontanel, occipital horns, wormian bones, decreased mineralization), skeletal survey in case of suspected fractures, evaluation of hip dysplasia (sub-luxation), ophthalmologic evaluation (corneal abnormalities, cataract), cardiac evaluation (ventricular septal defect, aortic root dilatation, pulmonary stenosis), kidney ultrasound (vesico-urinary reflux), liver function tests (associated liver involvement in glycosylation disorders), EEG (occasional association of epilepsy), BAEP (sensineural deafness in one patient), and brain MRI (migration defects). In the follow up, one should emphasize the importance of caloric intake, developmental assessment, physiotherapy and evaluation of possible osteoporosis to prevent frequent fractures in some of the ARCL II and GO patients.

Besides a detailed physical examination, skeletal survey, functional studies and imaging, it is essential to perform biochemical analysis (TIEF and Apo C-III IEF, repeated after 6 months of age) in all children with cutis laxa. The presence of the metabolic alteration has consequences both for organ involvement, prognosis assessment and counselling.

Until now no glycosylation disorder has been reported in patients with the phenotypically overlapping De Barsy and Gerodermia Osteodysplastica Syndromes. None of the DBS or GO patients was found so far carrying an ATP6VOA2 mutation. However, mutation in another Rab6-related protein involved in Golgi-trafficking was confirmed to underlie GO. ${ }^{23}$ One should consider the possibility of direct mutation analysis in CL Syndromes. Sequence analysis of FBLN4 and FBLN5 could be performed in clinically suspected ARCL type I. This type of cutis laxa can be readily distinguished from ARCL type II, because it is commonly associated with severe systemic involvement, cardiopulmonary lesions including infantile emphysema and supravalvular aortic stenosis. Molecular analysis of the ATP6VOA2 gene is so far the only possibility to evaluate the genetic background in ARCL II Syndrome. We hope that our review will serve as an inspiration for clinicians and geneticists to investigate patients with phenotypically overlapping syndromes as these may etiologically relate to other subunits of the V-ATPase complex.

\section{References}

1 Urban Z, Gao J, Pope FM, Davis EC: Autosomal dominant cutis laxa with severe lung disease: synthesis and matrix deposition of mutant tropoelastin. J Invest Dermatol 2005; 124: 1193-1199.

2 Morava E, Lefeber DJ, Urban Z et al: Defining the phenotype in an autosomal recessive cutis laxa syndrome with a combined congenital defect of glycosylation. Eur J Hum Genet 2008; 16: $28-35$.
3 Kornak U, Reynders E, Dimopoulou A et al: Impaired glycosylation and cutis laxa caused by mutations in the vesicular H+-ATPase subunit ATP6V0A2. Nat Genet 2008; 40: $32-34$.

4 Morava E, Wopereis S, Coucke P et al: Defective protein glycosylation in patients with cutis laxa syndrome. Eur J Hum Genet 2005; 13: 414-421.

5 Mehregan AH, Lee SC, Nabai H: Cutis laxa (generalized elastolysis): A report of four cases with autopsy findings. J Cut Path 1978; 5: 116-126.

6 Markova D, Zou Y, Ringpfeil F et al: Genetic heterogeneity of cutis laxa: a heterozygous tandem duplication within the fibulin-5 (FBLN5) gene. Am J Hum Genet 2003; 72: 998-1004.

7 Tassabehji M, Metcalfe K, Hurst J et al: An elastin gene mutation producing abnormal tropoelastin and abnormal elastic fibres in a patient with autosomal dominant cutis laxa. Hum Mol Genet 1998; 7: 1021-1028.

8 Van Maldergem L, Vamos E, Liebaers I et al: Severe congenital cutis laxa with pulmonary emphysema: a family with three affected sibs. Am J Med Genet 1988; 31: 455-464.

9 Kaler SG, Gallo LK, Proud VK et al: Occipital horn syndrome and a mild Menkes phenotype associated with splice site mutations at the MNK locus. Nat Genet 1994; 8: 195-202.

10 Moller LB, Tumer Z, Lund C et al: Similar splice-site mutations of the ATP7A gene lead to different phenotypes: classical Menkes disease or occipital horn syndrome. Am J Hum Genet 2000; 66: 1211-1220.

11 Donsante A, Tang J, Godwin SC et al: Differences in ATP7A gene expression underlie intrafamilial variability in Menkes disease/occipital horn syndrome. J Med Genet 2007; 44: $492-497$.

12 Patton MA, Tolmie J, Ruthnum P, Bamforth S, Baraitser M, Pembrey M: Congenital cutis laxa with retardation of growth and development. J Med Genet 1987; 24: 556-561.

13 Elahi E, Kalhor R, Banihosseini SS et al: Homozygous missense mutation in fibulin-5 in an Iranian autosomal recessive cutis laxa pedigree and associated haplotype. J Invest Dermatol 2006; 126: $1506-1509$.

14 Hucthagowder V, Sausgruber N, Kim KH, Angle B, Marmorstein LY, Urban Z: Fibulin-4: a novel gene for an autosomal recessive cutis laxa syndrome. Am J Hum Genet 2006; 78: $1075-1080$.

15 Kielty CM: Elastic fibres in health and disease. Expert Rev Mol Med 2006; 8: 1-23, . Review.

16 Claus S, Fischer J, Mégarbané H et al: A p.C217R mutation in fibulin-5 from cutis laxa patients is associated with incomplete extracellular matrix formation in a skin equivalent model. J Invest Dermatol 2008; 128: 1442-1450.

17 Reisner SH, Seelenfreund M, Ben-Bassat $M$ et al: Cutis laxa associated with severe intrauterine growth retardation and congenital dislocation of the hip. Acta Paediatr Scand 1971; 60: $357-360$.

18 Steiner CR, Cintra ML, Marques-de-Faria AP et al: Cutis laxa with growth and developmental delay, wrinkled skin syndrome and geroderma osteodysplastics: a report on two unrelated patients and a literature review. Genet Mol Biol 2005; 28: $181-190$.

19 Zlotogora J: Wrinkly skin syndrome and the syndrome of cutis laxa with growth and developmental delay represent the same disorder. Am J Med Genet 1999; 85: 194.

20 Kivuva EC, Parker MJ, Cohen MC, Wagner BE, Sobey G: De Barsy Syndrome: a review of the phenotype. Clin Dysmorphol 2008; 17: 99-107. Review.

21 Cantú JM, Sánchez-Corona J, Hernándes A, Nazará Z, García-Cruz D: Individualization of a syndrome with mental deficiency, macrocranium, peculiar facies, and cardiac and skeletal anomalies. Clin Genet 1982; 22: 172-179.

22 Rajab A, Kornak U, Budde BS et al: Geroderma osteodysplasticum hereditaria and wrinkly skin syndrome in 22 patients from Oman. Am J Med Genet A 2008; 146: 965-976. 
23 Hennies HC, Kornak U, Zhang H et al: Gerodermia osteodysplastica is caused by mutations in SCYL1BP1, a Rab-6 interacting golgin. Nat Genet 2008; 40: 1410-1412.

24 Vanakker OM, Martin L, Gheduzzi D et al: Pseudoxanthoma elasticum-like phenotype with cutis laxa and multiple coagulation factor deficiency represents a separate genetic entity. I Invest Derm 2007; 127: 581-587.

25 Hennekam RC: Costello Syndrome: an overview. Am J Med Genet $C$ Semin Med Genet 2003; 117C: 42-48, . Review.

26 Vaccaro M, Salpietro DC, Briuglia S, Merlino MV, Guarneri F, Dallapiccola B: Cutis laxa in Kabuki make-up syndrome. J Am Acad Dermatol 2005; 53: S247-S251.

27 Pinter R, Hogge WA, McPherson E: Infant with severe penicillamine embryopathy born to a woman with Wilson disease. Am J Med Genet A 2004; 128A: 294-298.

28 Armstrong L, Jimenez C, Hunter AG: A boy with developmental delay, malformations, and evidence of a connective tissue disorder: possibly a new type of cutis laxa. Am J Med Genet A 2003; 119A: 57-62.

29 Genevieve D, Baumann C, Huber C et al: A novel form of syndromic cutis laxa with facial dysmorphism, cleft palate, and mental retardation. J Med Genet 2004; 41: e77.

30 Shintaku M, Uemura Y, Fujii I et al: Neuroaxonal leukodystrophy associated with congenital cutis laxa: report of an autopsy case. Acta Neuropathol 2000; 99: 420-424.

31 Koklu E, Gunes T, Ozturk MA, Akcakus M, Buyukkayhan D, Kurtoglu S: Cutis laxa associated with central hypothyroidism owing to isolated thyrotropin deficiency in a newborn. Pediatr Dermatol 2007; 24: 525-528.

32 Biver A, De Rijcke S, Toppet V, Ledoux-Corbusier M, Van Maldergem L: Congenital cutis laxa with ligamentous laxity and delayed development, Dandy-Walker malformation and minor heart and osseous defects. Clin Genet 1994; 45: 318-322.

33 Jaeken J, Matthijs G: Congenital disorders of glycosylation: a rapidly expanding disease family. Annu Rev Genomics Hum Genet 2007; 8: 261-278, . Review.

34 Van Maldergem L, Yuksel-Apak M, Kayserili H et al: Cobblestonelike brain dysgenesis and altered glycosylation in congenital cutis laxa, Debre type. Neurology 2008; 71: 1602-1608.

35 van Reeuwijk J, Maugenre S, van den Elzen C et al: The expanding phenotype of POMT1 mutations: from Walker-Warburg Syndrome to congenital muscular dystrophy, microcephaly, and mental retardation. Hum Mutat 2006; 27: 453-459.

36 Martin PT: Dystroglycan glycosylation and its role in matrix binding in skeletal muscle. Glycobiology 2003; 13: 55R-66R.

37 Morava E, Willemsen MA, Wopereis S et al: High myopia and congenital myopathy with partial pachygyria in cutis laxa syndrome. Eur J Ophthalmol 2006; 16: 190-194.

38 De Barsey AM, Moens E, Dierckx L: Dwarfism, oligophrenia and degeneration of the elastic tissue in skin and cornea. A new syndrome? Helv Paediatr Acta 1968; 3: 305-313.
39 Qi J, Wang Y, Forgac M et al: The vacuolar (H+)-ATPase: subunit arrangement and in vivo regulation. J Bioenerg Biomembr 2007; 39: 423-426.

40 Guillard M, Dimopoulou A, Fischer B et al: Vacuolar $\mathrm{H}^{+}$-ATPase meets glycosylation in patients with cutis laxa. BBA 2009 (in press).

41 Hurtado-Lorenzo A, Skinner M, El Annan J et al: V-ATPase interacts with ARNO and Arf6 in early endosomes and regulates the protein degradative pathway. Nat Cell Biol 2006; 8: 124-136.

42 Nishi T, Forgac M: The vacuolar (H+)-ATPases-nature's most versatile proton pumps. Nat Rev Mol Cell Biol 2002; 3: 94-103.

43 Axelsson MA, Karlsson NG, Steel DM, Ouwendijk J, Nilsson T, Hansson GC: Neutralization of $\mathrm{pH}$ in the Golgi apparatus causes redistribution of glycosyltransferases and changes in the $\mathrm{O}$ glycosylation of mucins. Glycobiology 2001; 11: 633-644.

44 Freeze HH: Congenital disorders of glycosylation: CDG-I, CDG-II, and beyond. Curr Mol Med 2007; 7: 389-396, . Review.

45 Freeze HH: Genetic defects in the human glycome. Nat Rev Genet 2006; 7: 537-551.

46 Foulquier F, Ungar D, Reynders E et al: A new inborn error of glycosylation due to a Cog8 deficiency reveals a critical role for the Cog1-Cog8 interaction in COG complex formation. Hum Mol Genet 2007; 16: 717-730.

47 Foulquier F, Vasile E, Schollen E et al: Conserved oligomeric Golgi complex subunit 1 deficiency reveals a previously uncharacterized congenital disorder of glycosylation type II. Proc Natl Acad Sci USA 2006; 103: 3764-3769.

48 Morava E, Zeevaert R, Korsch E et al: A common mutation in the COG7 gene with a consistent phenotype including microcephaly, adducted thumbs, growth retardation, VSD and episodes of hyperthermia. Eur J Hum Genet 2007; 15: 638-645.

$49 \mathrm{Wu}$ X, Steet RA, Bohorov O et al: Mutation of the COG complex subunit gene COG7 causes a lethal congenital disorder. Nat Med 2004; 10: 518-523.

50 Stover EH, Borthwick KJ, Bavalia C et al: Novel ATP6V1B1 and ATP6V0A4 mutations in autosomal recessive distal renal tubular acidosis with new evidence for hearing loss. J Med Genet 2002; 39: 796-803.

51 Kornak U, Schulz A, Friedrich W et al: Mutations in the a3 subunit of the vacuolar $\mathrm{H}(+)$-ATPase cause infantile malignant osteopetrosis. Hum Mol Genet 2000; 9: 2059-2063.

52 Valayannopoulos V, Verhoeven NM, Mention K et al: Transaldolase deficiency: a new cause of hydrops fetalis and neonatal multi-organ disease. J Pediatr 2006; 149: 713-717.

53 Coman DJ: The congenital disorders of glycosylation are clinical chamelons. Eur J Hum Genet 2008; 16: 2-4.

54 Holbrook KA, Byers PH: Structural abnormalities in the dermal collagen and elastic matrix from the skin of patients with inherited connective tissue disorders. J Invest Dermatol 1982; 79 (Suppl 1): 7s-16s. 